\title{
Finite Larmor Radius Effects on the Magnetorotational Instability
}

\author{
Nathaniel M. Ferraro \\ Princeton Plasma Physics Laboratory, Princeton, NJ, 08543-0451
}

\begin{abstract}
The linear dispersion relation for the magnetorotational instability (MRI) is derived including finite Larmor radius (FLR) effects. In particular, the Braginskii form of the ion gyroviscosity, which represents the first-order FLR corrections to the two-fluid equations, is retained. It is shown that FLR effects are the most important effects in the limit of weak magnetic fields, and are much more important than the Hall effect when $\beta_{i} \gg 1$, where $\beta_{i}$ is the ratio of the ion thermal pressure to the magnetic pressure. FLR effects may completely stabilize even MRI modes having wavelengths much greater than the ion Larmor radius. Some implications for astrophysical accretion disks are discussed.
\end{abstract}

Subject headings: accretion, accretion disks - instabilities - MHD — plasmas

\section{Introduction}

The magnetorotational instability is a local instability which may be present in accretion disks having sheared azimuthal flow and a weak magnetic field (Balbus \& Hawley 1991). Turbulence resulting from this instability is thought to play an important role in the radial transport of angular momentum in such systems (Balbus \& Hawley 1998). Here we explore the modifications to the local, two-fluid theory of the MRI in the linear regime due to finite Larmor radius (FLR) effects.

Rosenbluth et al. (1962) have shown using kinetic theory that FLR effects can be stabilizing to "weakly unstable" modes - defined as modes having a linear growth rate much smaller than the ion cyclotron frequency - even when the mode wavelength is much larger than the ion Larmor radius. It was later shown by Roberts \& Taylor (1962) that this result could be obtained from fluid theory by retaining the gyroviscous stress component of the ion pressure tensor. In most physical scenarios the MRI is weakly unstable in the sense of Rosenbluth et al., and indeed we show that the MRI may be completely stabilized by gyroviscous effects at scales much larger than the ion Larmor radius. In some cases this stabilization significantly constrains the spectrum of linearly unstable modes.

The effect of the Hall term, which accounts for differences between the electron and ion fluid velocities, has been examined previously by Wardle (1999), Balbus \& Terquem (2001), Salmeron \& Wardle (2003), and Krolik \& Zweibel (2006). In particular, it was found that the Hall effect may be either stabilizing or destabilizing, depending on whether the equilibrium magnetic field is aligned or anti-aligned to the equilibrium angular velocity. It was also found that the Hall effect is important only when the ion cyclotron frequency is comparable to, or smaller than, the orbital frequency. This situation may occur in early galaxy formation where the magnetic fields are still weak, or in weakly ionized protostellar disks. Krolik \& Zweibel (2006) have suggested that, in this limit, short-wavelength modes are likely suppressed by viscous or resistive damping, leaving only slowly growing, long-wavelength modes as the magnetic field get sufficiently weak. However, their analysis is restricted to low- $\beta_{i}$ plasmas as they do not consider FLR effects, which we show to be much more important than the Hall effect in the weakfield limit. The strong FLR stabilization of the MRI in the weak-field limit may have important implications for the possible role of the MRI in the amplification of weak, primordial magnetic fields.

The gyroviscous stress is defined as the traceless, perpendicular part of the ion stress tensor which does not depend explicitly on the collision 
frequency (Ramos 2005). In typical cases, this stress arises primarily from variations in particle drift velocities over the scale of a Larmor orbit (Kaufman 1960). However, other effects may contribute to this stress, including gradients in heat fluxes. Braginskii's form of the ion gyroviscous stress is appropriate for collisional plasmas (in the sense that the ion mean-free-path is small compared to the hydrodynamic perturbation lengthscale), and in the limit where the ion cyclotron period is short compared to collisional and hydrodynamic time-scales (Braginskii 1965). More general, but more complicated, expressions for the gyroviscous force have been derived, which are applicable to a broader range of collisionality regimes and dynamical time scales; Ramos (2005) and references therein provide derivations and discussions of these alternate forms. We choose to work with Braginskii's form here because it is (relatively) simple and applies to a broad range of astrophysical objects.

The MRI in the collisionless regime, where the collisional mean-free-path is greater than the mode wavelength, has been explored by Quataert et al. (2002) and Sharma et al. (2003) using kinetic closures. Islam \& Balbus (2005) have extended the single-fluid MHD treatment to lower collisionality regimes by including the Braginskii (1965) form of the parallel viscosity, and have obtained results similar to those obtained using kinetic closure. These various analyses have found the linear growth rate to be enhanced by a factor of order unity at lower collisionality when an azimuthal component of the magnetic field is present, but the criterion for instability was found not to differ from the MHD result. We do include the parallel viscous stress for completeness in our analysis, as formally it may be larger than the gyroviscous stress. However, for the sake of simplicity we restrict the MRI mode wavevector and magnetic field to be normal to the accretion disk, in which case the parallel viscosity has no effect on the MRI. This case is the most unstable one in the collisional limit, which is the limit in which we are mainly interested.

\section{Linear Theory}

\subsection{Model}

We consider the two-fluid MHD equations:

$$
\begin{aligned}
\frac{\partial n}{\partial t}= & -\nabla \cdot(n \vec{v}) \\
n \frac{\partial \vec{v}}{\partial t}= & -n \vec{v} \cdot \nabla \vec{v}+\frac{\vec{J} \times \vec{B}}{c}- \\
& -\nabla p-\nabla \cdot \Pi-n g(r) \hat{r} \\
\frac{\partial \vec{B}}{\partial t}= & -c \nabla \times \vec{E}
\end{aligned}
$$

where

$$
\begin{gathered}
\vec{E}=-\frac{1}{c} \vec{v} \times \vec{B}+\frac{1}{n e}\left(\frac{1}{c} \vec{J} \times \vec{B}-\nabla p_{e}\right) \\
\vec{J}=\frac{c}{4 \pi} \nabla \times \vec{B} ; \quad p=p_{i}+p_{e} .
\end{gathered}
$$

The terms representing two-fluid effects are the Hall term $(\vec{J} \times \vec{B} /$ nec $)$ and the electron pressure gradient in the definition of $\vec{E}$. We assume barytropic pressure variations of the form

$$
d p_{s}=\Gamma T_{s} d n
$$

for each species $s$ where, for example, $\Gamma=5 / 3$ for an adiabatic equation of state. For $\Pi$ we use the leading order terms in the Braginskii (1965) closure:

$$
\Pi=\Pi^{v}+\Pi^{g v},
$$

where

$$
\begin{aligned}
\Pi^{v}= & \eta_{0} \frac{p_{i}}{2 \nu_{i}}(\mathrm{I}-3 \hat{b} \hat{b})(\hat{b} \cdot \mathrm{W} \cdot \hat{b}) \\
\Pi^{g v}= & \frac{p_{i}}{4 \omega_{c i}}\{\hat{b} \times \mathrm{W} \cdot(\mathrm{I}+3 \hat{b} \hat{b})+ \\
& \left.+[\hat{b} \times \mathrm{W} \cdot(\mathrm{I}+3 \hat{b} \hat{b})]^{\top}\right\}
\end{aligned}
$$

where $\hat{b}=\vec{B} / B, \omega_{c i}=e B_{z 0} / m_{i} c$ is the ion cyclotron frequency, $\nu_{i}$ is the ion collision frequency, and the coefficient $\eta_{0} \approx 0.96$ is a factor determined by kinetic analysis (Braginskii 1965). The rate-ofstrain tensor is

$$
\mathrm{W}=\nabla \vec{v}+(\nabla \vec{v})^{\top}-\frac{2}{3} \mathrm{I} \nabla \cdot \vec{v} .
$$

$\Pi^{v}$ is the parallel viscosity term considered by Balbus (2004) and Islam \& Balbus (2005), which represents the lowest-order in $\partial_{t} / \nu_{i}$ correction to the fluid equations. (It will be shown, however, 
that the parallel viscosity plays no role in the MRI in the configuration we choose to examine.) $\Pi^{g v}$ is the gyroviscous force, which represents the lowest-order FLR correction to the fluid equations. This form of $\Pi$ is appropriate in the limit where $\omega_{c i} \gg \nu_{i}$. Together with the short mean-free-path condition $\left(k_{\|} \lambda_{m f p} \ll 1\right.$, where $\left.\lambda_{m f p} \sim v_{t} / \nu_{i}\right)$ necessary to justify the fluid closure, this restricts the validity of our results to the parameter regime where

$$
k_{\|} v_{t} \ll \nu_{i} \ll \omega_{c i} .
$$

In the case of an accretion disk where $k_{\|} \gtrsim 1 / d$ is limited by the disk height $d \sim v_{t} / \Omega$, this validity condition becomes $\Omega \ll \nu_{i} \ll \omega_{c i}$.

\subsection{Equilibrium}

We consider a cylindrical equilibrium, using the standard coordinates $(r, \theta, z)$, and assume axisymmetry in the azimuthal coordinate $\left(\partial_{\theta}=0\right)$. For simplicity, we assume the equilibrium magnetic field is of the form $\vec{B}_{0}=B_{z 0} \hat{z}$, and that the equilibrium fluid velocity is of the form $\vec{v}_{0}=r \Omega(r) \hat{\theta}$. Without loss of generality, we orient our coordinate system so that $\Omega(r)>0$ at the radius of interest. For such a configuration, radial force balance is satisfied when

$$
g(r)=r \Omega^{2}(r)+\frac{\rho_{i}^{2} \omega_{c i}}{2 r^{2}} \frac{\partial}{\partial r}\left[r^{3} \Omega^{\prime}(r)\right],
$$

where $\rho_{i}=v_{t} / \omega_{c i}$ is the ion Larmor radius, and $v_{t}=\sqrt{T_{i} / m_{i}}$ is the ion thermal velocity. We neglect any equilibrium structure in the $z$-direction. In the following derivation, we choose to use equation (7) to eliminate $g(r)$ in favor of $\Omega(r)$ (hereafter we will drop the explicit dependence of $\Omega$ on $r)$.

\subsection{Local Linear Dispersion Relation}

We consider linear perturbations about this equilibrium having scale lengths $1 / k$ much smaller than the equilibrium flow gradient scale length $L=\Omega / \Omega^{\prime}$, so that $\delta=1 /|k L| \ll 1$. In this limit, we may assume that the normal modes of the system are plane waves to lowest order in $\delta$. We restrict our analysis to perturbations $\propto e^{i(k z-\omega t)}$ for simplicity, as this is the most unstable case in both the MHD and collisionless limits (Quataert et al. 2002). Carrying out the linearization of equations (1) yields the following dispersion relation, to lowest order in $\delta$ :

$$
\begin{aligned}
0= & \left(W^{2}+W \frac{8 i \eta_{0}}{3} \frac{\omega_{c i}}{\nu_{i}} A-C\right) \times \\
& \times\left\{W^{4}+a_{2} W^{2}+a_{0}\right\}
\end{aligned}
$$

where

$$
\begin{aligned}
a_{2}= & -2(2+R+K)+ \\
& +2 A(4+R-2 A)-H(R+H) \\
a_{0}= & {[K-A(R+2 H)+2(R+H)] \times } \\
& \times[K-A(R+2 H)+H(2+R)] .
\end{aligned}
$$

We define the dimensionless quantities

$$
\begin{aligned}
W & =\omega / \Omega \\
K & =\left(v_{A} k / \Omega\right)^{2} \\
A & =K \beta_{i}\left(\Omega / \omega_{c i}\right) / 4 \equiv K F \\
H & =K\left(\Omega / \omega_{c i}\right) \\
C & =\left(c_{s} k / \Omega\right)^{2} \\
R & =r \Omega^{\prime} / \Omega
\end{aligned}
$$

and characteristic velocities $v_{A}^{2}=B_{z 0}^{2} / 4 \pi n_{0} m_{i}$ and $c_{s}^{2}=\Gamma\left(T_{e}+T_{i}\right) / m_{i}$. Here $\beta_{i}=8 \pi p_{i 0} / B_{0}^{2}$ is the ratio of ion thermal pressure to magnetic pressure. The dimensionless parameter $A$ measures the importance of the gyroviscous force, and setting $A=0$ is equivalent to omitting $\nabla \cdot \Pi^{g v}$ in the ion force equation. Similarly, $H$ measures the importance of the Hall term in Ohm's law, and $K$ measures the importance of magnetic tension. $R$ is the ratio of the radial coordinate to the equilibrium flow gradient scale length, and is taken to be $\sim \mathcal{O}(1)$. For a Keplerian disk, $R=-3 / 2$. For convenience we have also defined

$$
F=\frac{\beta_{i}}{4} \frac{\Omega}{\omega_{c i}},
$$

which is the ratio of the gyroviscous force to the magnetic tension force.

Equation (8) contains two uncoupled modes. The first factor contains the acoustic mode, which may be damped by the parallel viscosity when $A \neq 0$, and is not of interest here. (While $\omega_{c i} / \nu_{i}$ is generally large, $A \omega_{c i} / \nu_{i} \sim C \Omega / \nu_{i} \ll C$ in the collisional regime, so the effect on the acoustic mode is small.) The second factor, enclosed in braces, contains the MRI. In the limit where $A \rightarrow 0$ (no FLR effects), $H \rightarrow 0$ (no Hall effect), and $\Omega / \nu_{i} \rightarrow 0$ 

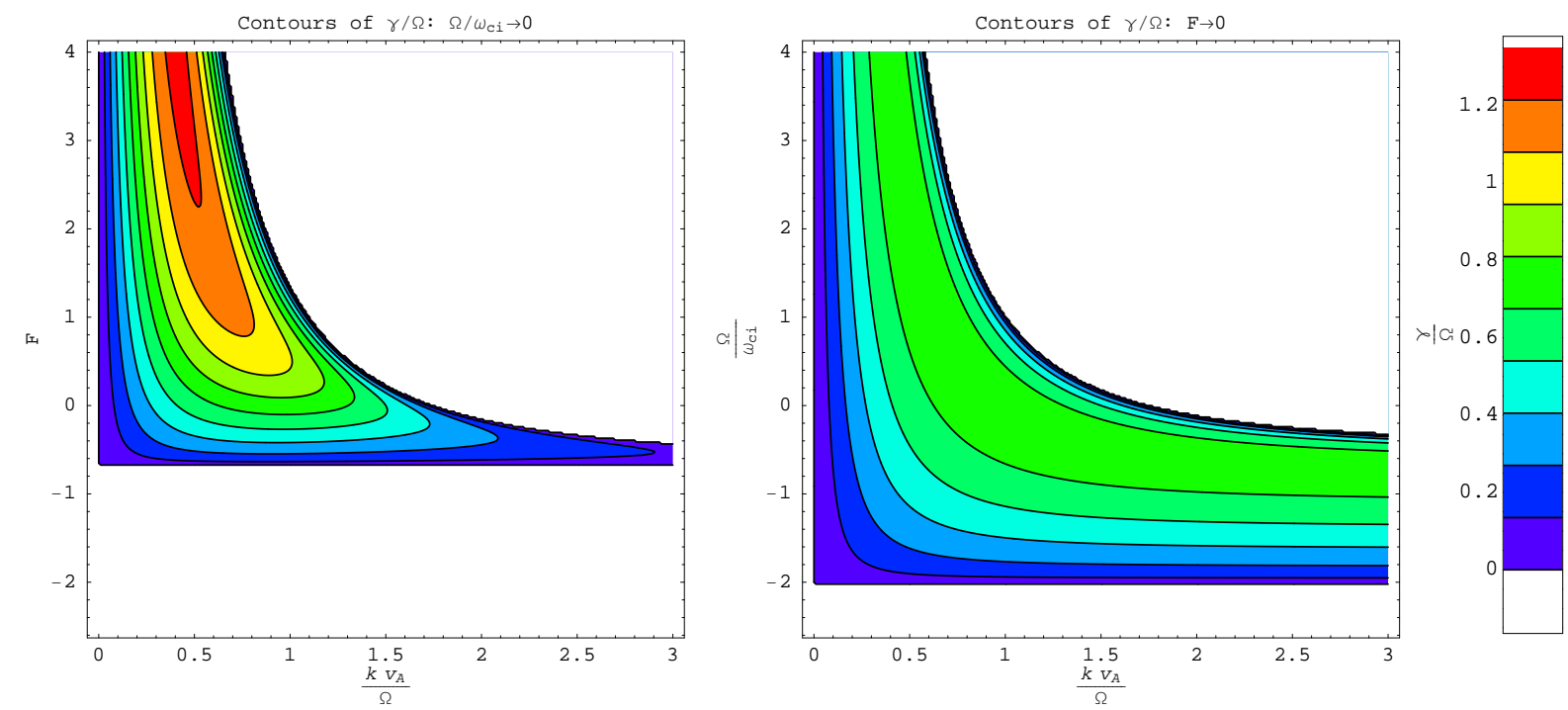

Fig. 1. - Contours of the growth rate of the unstable solution to equation (8) are plotted versus $k v_{A} / \Omega$. Left: The growth rate is plotted for various values of $F$, which measures the importance of FLR effects, in the limit where $\Omega / \omega_{c i} \ll 1$ (no Hall effect). $F=0$ is the ideal MHD result. Right: The growth rate is plotted for various values of $\Omega / \omega_{c i}$, which measures the importance of the Hall effect, in the limit where $F \ll 1$ (no FLR effects). $\Omega / \omega_{c i}=0$ is the ideal MHD result.

(collisional regime), the dispersion relation of Balbus \& Hawley (1998) is recovered. Note that the parallel viscosity $\left(\propto \eta_{0}\right)$ affects only the acoustic mode and not the MRI. Evidently, there is no $\mathcal{O}\left(\Omega / \nu_{i}\right)$ correction to the MHD result for the MRI when $\vec{B}_{0}=B_{z 0} \hat{z}$, which is in agreement with the findings of Sharma et al. (2003) and Islam \& Balbus (2005). Here we are interested in corrections to the collisional mode, for which the $\vec{B}_{0}=B_{z 0} \hat{z}$ case is the most unstable. Extending this analysis to a more general magnetic field configuration substantially complicates the analysis.

It should also be noted that $\omega_{c i}$ is a signed quantity since it is proportional to $B_{z}$, which may be positive or negative. Since we have chosen the coordinate system so that $\Omega$ is positive, $\operatorname{sign} \omega_{c i}=\operatorname{sign} \vec{B} \cdot \vec{\Omega}=\operatorname{sign} F$. It has been shown previously that the effect of the Hall term on the MRI depends strongly on the sign relative signs of $\omega_{c i}$ and $\Omega$ (Wardle 1999). The effect of the gyroviscous force has a similar dependence.

The growth rate $\gamma=\operatorname{Im} \omega$ of the unstable solution to equation (8) is plotted in figure 1 for a Keplerian rotation profile $(R=-3 / 2)$. Note that the abscissa should be read as a normalized wavenum- ber and not a normalized magnetic field strength, because $F$ and $\Omega / \omega_{c i}$ are dependent on $B$. When $\omega_{c i}>0$, and hence $F>0$ also, both the FLR and the Hall effects can be seen to move the most unstable mode to lower wavenumbers, and to reduce the value of $K$ at which the MRI is completely stabilized. Also, FLR effects increase the growth rate of the most unstable mode. When $\omega_{c i}<0$, and hence $F<0$, both effects are seen instead to increase the cutoff value of $K$ all the way to the point where modes of any wavelength for which this analysis is valid are unstable. Presumably, the inclusion of a finite resistivity would damp this resonance, as it does in the case of the resonance due to the Hall effect (Balbus \& Terquem 2001). When $F$ or $\Omega / \omega_{c i}$ becomes sufficiently negative $\left(F<-2 / 3\right.$ when $\Omega / \omega_{c i} \rightarrow 0$, or $\Omega / \omega_{c i}<-2$ when $F \rightarrow 0$ ), all values of $k$ are suddenly completely stabilized. (It has been shown by Balbus \& Terquem (2001) that this stabilization is less sudden when finite resistivity is included.)

\subsection{Instability Criterion}

Applying the Routh-Hurwitz theorem to equation (8), we find that the condition for stability of 
an MRI mode is that:

$$
a_{2}<0 \text { and } a_{0}>0
$$

This criterion is highly complicated, and for general values of $A$ and $H$, there may be multiple stable and unstable regions in $K$-space.

In the ideal limit, when $A \rightarrow 0$ and $H \rightarrow 0$, the instability criterion of Balbus \& Hawley (1998), $K<-2 R$, is recovered. This limit is well understood, and in this case stabilization at high- $K$ is due to the effect of magnetic tension. In this limit, instability does not exist in flows in which the angular velocity increases with radius $(R>0)$.

The limit $A \rightarrow 0$, in which the Hall effect is dominant over the FLR effects, has also been considered before. In this limit, equation (8) reduces to the dispersion relation of Balbus \& Terquem (2001). Since $A / H=\beta_{i} / 4$, this limit describes accretion disks having $\beta_{i} \ll 1$. Formally, the instability criterion in this case remains somewhat complicated because the signs and relative magnitudes of most of the terms are undetermined in general. There is some discussion of the instability criterion in this case by Wardle (1999) and Balbus \& Terquem (2001), as well as insight into its physical meaning. We will not repeat this discussion, except to mention a few interesting points. The first is that there may exist some values of $\Omega / \omega_{c i}$ for which modes of any wavelength are unstable (this is true in the Keplerian case for $\left.-2<\Omega / \omega_{c i}<-1 / 2\right)$. Also, some unstable modes may be present in disks in which angular velocity increases with radius $(R>0)$, in contrast to the ideal result (Balbus \& Terquem 2001).

We are more interested in the opposite limit, $\beta_{i} \gg 1$, in which FLR effects are dominant over the Hall effect. Taking $H \rightarrow 0$, the dispersion relation for the MRI reduces to

$$
\begin{aligned}
0= & W^{4}- \\
& -2[2+R+K-A(4+R-2 A)] W^{2}+ \\
& +(K-A R+2 R)(K-A R)
\end{aligned}
$$

and the criterion for instability is found to be

$$
K<-\frac{2 R}{1-F R}
$$

For the usual case where $R<0$, all modes are completely stabilized if $F<-1 /|R|$. As with the
Hall effect, gyroviscosity allows unstable modes to exist when $R>0$; in this case, unstable modes may exist when $F>1 / R$. In the limit where the gyroviscous force dominates the force of magnetic tension, $F \gg 1$, the instability criterion becomes simply

$$
A<2 \text { and } F>0
$$

or, equivalently, $\left(k \rho_{i}\right)^{2}<4 \Omega / \omega_{c i}$, In the case where $F<0$, there are no unstable modes in this limit.

\section{Discussion and Conclusions}

The gyroviscous stress arises from changes to the guiding-center drifts due to the gradients of magnetohydrodynamic forces (the electric field, in this case) across a gyro-orbit. A more complete and quantitative discussion of this effect is discussed by Kaufman (1960). Due to their larger Larmor radius, the ions are more affected by this modification that the electrons, leading to the generation of currents. Essentially, the stabilization (or destabilization) of the MRI by FLR effects is due to the currents generated by the gyroviscous force being out of phase (or in phase) with the current of the ideal-MHD MRI eigenmode.

The effect of the gyroviscous stress becomes relatively more important to the behavior of the MRI as the magnetic field strength is decreased. The relative importance of this stress to the magnetic tension is $A / K=F \propto B^{-3}$, and the relative importance of the gyroviscous stress to the Hall effect is $A / H=\beta_{i} / 4 \propto B^{-2}$. Note that this means analyses of the MRI in the "weak-field" limit (in the sense that $\Omega / \omega_{c i} \gtrsim 1$ ) which did not consider FLR effects are valid only for $\beta_{i} \ll 1$. If the magnetic field is sufficiently weak, the validity condition for the Braginskii closure, equation (6), may be violated since $k v_{t} / \omega_{c i}=\sqrt{2 H F} \propto B^{-1}$, in which case the above analysis no longer strictly applies. Of course, gyroviscosity does not shut off at this point; indeed, FLR effects are expected to become increasingly powerful as the magnetic field is decreased further, though not in a way that is correctly described by equation (4). Therefore it is probable that the low- $K$ modes which survive at the lowest magnetic field strengths covered by this analysis will be completely stabilized as the magnetic field decreases further. This is the proper resolution to the inconsistency of the ideal MHD 
result than the MRI remains unstable as $B \rightarrow 0$ in the non-dissipative case. Because the MRI is not present at arbitrarily low magnetic fields, its role in the amplification of primordial astrophysical magnetic fields is severely restricted.

It should also be noted that FLR effects may completely stabilize MRI modes having wavelengths much greater than the ion Larmor radius, where $k \rho_{i} \ll 1$. As was shown by Rosenbluth et al. (1962), this is possible for "weakly unstable" modes like the MRI. Restricting our analysis to the FLR-dominated limit $(F \gg 1)$, MRI modes are stabilized when $\left(k \rho_{i}\right)^{2}>4\left(\Omega / \omega_{c i}\right)$, where $\Omega / \omega_{c i}$ is typically small in astrophysical accretion disks. This stabilization may be made more important by the fact that in an accretion disk the lower bound on $k$ is set by the height of the disk $d$, which may be much smaller than the equilibrium flow gradient scale length $L$. Although a proper understanding of this phenomenon must take into account the $z$-stratification of the disk equilibrium, we may estimate that the smallest wavenumber present in the disk is $\sim \pi / d$. The criterion for complete stabilization by FLR effects of all MRI modes within an accretion disk at some distance from the central mass is then

$$
\pi^{2}\left(\rho_{i} / d\right)^{2} \gtrsim 4 \Omega / \omega_{c i}
$$

In the typical case where $d \sim v_{t} / \Omega$ this inequality reduces to $\omega_{c i} / \Omega \lesssim \pi^{2} / 4$. While this is not typically satisfied in astrophysical accretion disks, it may be satisfied in nascent galaxies with weak magnetic fields, or weakly ionized protostellar disks (Krolik \& Zweibel 2006). It is important to recall here that the condition for validity of our analysis for wavelengths of this scale requires $\Omega \ll \omega_{c i}$; whether FLR effects are more or less stabilizing than our result would predict outside this range of validity is a matter for further research.

For a concreteness, we consider the example of an active galactic nucleus (AGN) in a weak magnetic field, for which the conditions for validity of the Braginskii equations are met, and in which the effect of the gyroviscous force is particularly strong. We assume Keplerian rotation $(R=-3 / 2)$, a central mass of $10^{7} M_{\odot}, B=10$ $\mathrm{nG}, n=10 \mathrm{~cm}^{-3}$, and $T_{i}=1 \mathrm{eV}$, at a distance of $10^{16} \mathrm{~cm}$. For this case, the relevant dimensionless parameters are $F \approx 384, H \approx 3.81 \times 10^{-4}$, and

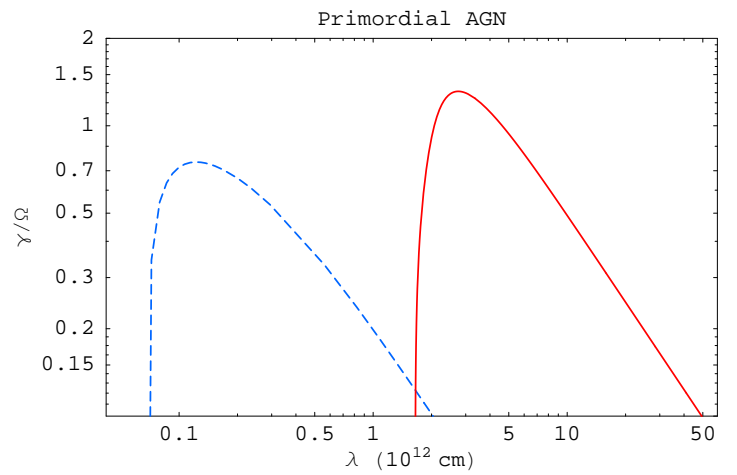

Fig. 2.- The growth rate of the MRI is plotted versus wavelength for a Keplerian, $10^{7} M_{\odot}$ active galactic nucleus in a weak magnetic field $(B=10 \mathrm{nG})$, at a distance $10^{16} \mathrm{~cm}$. The solutions to the dispersion relation including (solid line), and excluding (broken line) the FLR correction are plotted. Gyroviscosity completely stabilizes the shorter-wavelength modes, and enhances the longer-wavelength modes.

$K \approx 1.96 \times 10^{-5}$ at $k=\Omega / v_{t}$. The growth rates of the MRI under these conditions are plotted in figure 2. From that figure it can be seen that in both cases the FLR correction increases the wavelength of the fastest-growing mode from $\approx 10^{11}$ $\mathrm{cm}$ to $\approx 25 \times 10^{11} \mathrm{~cm}$, and similarly increases the cutoff wavelength below which there are no unstable modes. In this example, the weakness of the magnetic field is crucial to the importance of the FLR effect. If the magnetic field strength is raised by two orders of magnitude, the FLR correction becomes insignificant. Therefore, "primordial" accretion disks in weak magnetic fields will be most affected by the FLR correction, whereas disks in stronger fields $(B \geq 10 \mu \mathrm{G})$ may be completely unaffected, unless they are unusually hot and dense, or have an orbital frequency not much smaller than the ion cyclotron frequency.

The nonlinear saturation of the MRI cannot be properly addressed by linear analysis. Because gyroviscosity is not dissipative, and there is no energy associated with the gyroviscous term, one might expect that the ultimate nonlinear saturated state should not be affected strongly by the gyroviscosity. Ultimately, questions of nonlinear saturation should be addressed by numerical sim- 
ulation.

The author is grateful for helpful discussions with Dr. Gregory Hammett and Ian Parrish.

\section{REFERENCES}

Balbus, S. A. 2004, ApJ, 616, 857

Balbus, S. A. \& Hawley, J. F. 1991, ApJ, 376, 214

—. 1998, Rev. Mod. Phys., 70, 1

Balbus, S. A. \& Terquem, C. 2001, ApJ, 552, 235

Braginskii, S. I. 1965, in Reviews of Plasma Physics, ed. M. A. Leontovich, Vol. 1 (New York: Consultants Bureau), 205-311

Islam, T. \& Balbus, S. 2005, ApJ, 633, 328

Kaufman, A. N. 1960, Phys. Fluids, 3, 610

Krolik, J. H. \& Zweibel, E. G. 2006, ApJ, 644, 651

Quataert, E., Dorland, W., \& Hammett, G. 2002, ApJ, 577, 524

Ramos, J. J. 2005, Phys. Plasmas, 12, 112301

Roberts, K. V. \& Taylor, J. B. 1962, Phys. Rev. Lett., 8, 197

Rosenbluth, M. N., Krall, N. A., \& Rostoker, N. 1962, Nucl. Fusion Suppl., 1, 143

Salmeron, R. \& Wardle, M. 2003, MNRAS, 345, 992

Sharma, P., Hammett, G. W., \& Quataert, E. 2003, ApJ, 596, 1121

Wardle, M. 1999, MNRAS, 307, 849

This 2-column preprint was prepared with the AAS LATEX macros v5.2. 